\title{
The efficacy and effectiveness of drugs for diabetes: how do clinical trials and the real world compare?
}

\author{
Richard E. Pratley
}

Received: 23 April 2014 / Accepted: 28 April 2014 /Published online: 22 May 2014

(C) Springer-Verlag Berlin Heidelberg 2014

Keywords Comparative effectiveness research .

DPP-4 inhibitor · Drug efficacy · Observational study ·

Randomised controlled trial $\cdot$ Sulfonylurea $\cdot$ Type 2 diabetes

\section{Abbreviation \\ RCT Randomised controlled trial}

For many years, the randomised controlled clinical trial (RCT) has been the gold standard for evaluating the efficacy, tolerability and safety of drugs. The modern framework for RCTs was developed in the mid-20th century through the leadership of the UK Medical Research Council and the US National Institutes of Health in collaboration with academic clinical researchers and scientists, among others [1]. Adoption of the RCT by regulatory agencies, including the US Food and Drug Administration (FDA) and the European Medicines Agency, as a requirement for the approval of most new drugs significantly shifted the clinical trial landscape. Today, the majority of patients who participate in RCTs do so in studies sponsored by pharmaceutical and device companies. The advantages of RCTs are well known to most clinical researchers and clinicians. Random assignment to treatment minimises allocation bias, that is, the chance that known and unknown systematic

\footnotetext{
R. E. Pratley $(\bowtie)$

Florida Hospital Diabetes Institute, Orlando, FL, USA

e-mail: Richard.Pratley@flhosp.org

R. E. Pratley

Translational Research Institute for Metabolism and Diabetes,

Orlando, FL, USA

R. E. Pratley

Sanford|Burnham Medical Research Institute, 2415 North Orange

Avenue, Orlando, FL 32804, USA
}

differences between treatment groups affect the outcomes. Masking of treatment assignment when possible ensures that any conscious and unconscious biases of investigators and participants do not alter outcomes. Finally, analysing responses according to treatment assignment (the intention-totreat principle) minimises the chance that differential followup biases the results.

Despite the benefits of RCTs, there are limitations to this approach. In particular, patients in RCTs often do not mirror the general population with the disease under study. To address regulatory requirements in drug and device trials, the inclusion and exclusion criteria are often narrowly defined. For example, a trial may specify that only patients with type 2 diabetes between the ages of 18 and 70 on metformin at a dose of at least 1,500 mg with an $\mathrm{HbA}_{1 \mathrm{c}}$ level between $7 \%$ and $10 \%$ who have not had a recent myocardial infarction may be included. While this provides robust information about a specific segment of the population, it limits the extent to which the results can be generalised to the broader population. To address this limitation, drug and device companies often conduct multiple trials, each targeting a different patient segment. However, this increases costs and may create issues when pooling the data. Other, less apparent, limitations also may affect the extent to which the results of RCTs can be generalised to a broader population. For example, patients who volunteer to participate in clinical trials are often healthier than the general population with disease. Moreover, the nature of the clinical trials process tends to select patients who are more highly motivated - they often join a clinical trial to improve their health status and may be more compliant with other aspects of their care. The consent process, with its thorough discussions of the benefits and risks of treatment, creates a more informed patient who may be more likely to adhere to therapy. Finally, frequent contact with the research coordinator and principal investigator emphasise and reinforce the need for adherence. All these factors, and more, create a 
situation quite unlike clinical practice. Thus, while RCTs can provide robust evidence of the efficacy of an intervention, they do not inform us about the effectiveness of the intervention in clinical practice. To address this knowledge gap, more 'real-life' clinical effectiveness trials are being planned and executed.

In this issue of Diabetologia, Ahrén and colleagues have compared the efficacy of sulfonylureas and the dipeptidyl peptidase-IV (DPP-4) inhibitor vildagliptin in RCTs with the clinical effectiveness of these drugs in an observational 'reallife' study in patients with type 2 diabetes [2]. The efficacy of the treatments was determined by analysing pooled 24-week data from five randomised, double-blind, controlled trials comparing vildagliptin (50 mg once or twice a day, $n=2,788$ ) with glimepiride (up to $6 \mathrm{mg} /$ day, $n=1,259$ ) or gliclazide (up to $320 \mathrm{mg} /$ day, $n=433$ ). All patients were treated with background metformin therapy at doses $\geq 1,500 \mathrm{mg} /$ day. The clinical effectiveness of the treatments was compared in a subset of patients drawn from the Effectiveness of Diabetes control with vildaGliptin and vildagliptin/mEtformin (EDGE) study, a large, nonrandomised, open-label, clinic-based observational study [3]. In this study, treatment, clinic visits and $\mathrm{HbA}_{1 \mathrm{c}}$ monitoring were at the discretion of the physician. Patients with type 2 diabetes on background metformin therapy received vildagliptin (at the standard dosage of $50 \mathrm{mg}$ twice a day, $n=7,002$ ) or sulfonylureas (at a dose determined by the clinician, $n=3,702$ ). The groups in the RCT and observational studies were quite similar in terms of age (57 years), duration of diabetes (just over 5 years) and the proportion of men (54\%). The mean baseline $\mathrm{HbA}_{1 \mathrm{c}}$ was higher in the observational study $(\sim 8.3 \%)$ than in the pooled RCT population (7.9\% and $7.6 \%$ for the groups randomised to vildagliptin and sulfonylureas, respectively). In the population as a whole, the change in $\mathrm{HbA}_{1 \mathrm{c}}\left(\Delta \mathrm{HbA}_{1 \mathrm{c}}\right)$ was correlated to the baseline $\mathrm{HbA}_{1 \mathrm{c}}\left(r^{2}=0.36\right)$ as one would expect, whereas other clinical variables including age, body weight and sex explained very little of the response. Since the RCT and observational populations started from different mean $\mathrm{HbA}_{1 \mathrm{c}}$ levels, $\Delta \mathrm{HbA}_{1 \mathrm{c}}$ as a function of baseline $\mathrm{HbA}_{1 \mathrm{c}}$ was compared between treatments and populations. For sulfonylurea treatment, the slope of the curve of $\Delta \mathrm{HbA}_{1 \mathrm{c}}$ and baseline $\mathrm{HbA}_{1 \mathrm{c}}$ was steeper in the observational study than in the pooled RCT data, and the interaction term was significant. Thus, patients in the observational study had a smaller $\Delta \mathrm{HbA}_{1 \mathrm{c}}$ at a lower baseline $\mathrm{HbA}_{1 \mathrm{c}}$ than did those in the RCTs, while at higher baseline $\mathrm{HbA}_{1 \mathrm{c}}$ levels, the responses tended to converge, although always remaining inferior to those of the RCT population. In contrast, for vildagliptin treatment, the slopes of the $\Delta \mathrm{HbA}_{1 \mathrm{c}}$ vs baseline curves were virtually superimposable and the interaction term was non-significant. The authors speculate that hypoglycaemia (or fear of hypoglycaemia) may have limited dosing of sulfonylureas and, thus, efficacy in the observational study where dosing was not specified by protocol. This may have been particularly true in those patients with a low baseline $\mathrm{HbA}_{1 \mathrm{c}}$. In contrast, because vildagliptin is associated with a low rate of hypoglycaemia when used in combination with metformin, there was no need (or perceived need) to limit dosing, and therefore efficacy and effectiveness were similar. Unfortunately, in the observational study, the occurrence of hypoglycaemia and the doses of medications were not routinely recorded. Thus, it is impossible to answer this important question.

What does this analysis teach us beyond the comparative efficacy/effectiveness of two diabetes treatments? First, the populations in RCTs and observational studies are likely to differ in important ways that make direct comparisons difficult. In the paper by Ahrén et al, there were clinically relevant differences in baseline $\mathrm{HbA}_{1 \mathrm{c}}$, for example [2], suggesting that the RCT population may have been healthier. Creative and non-traditional approaches to understanding the data in such circumstances may be required and will likely differ from analysis to analysis. Second, the nature of observational studies limits the data available for answering questions raised by the analyses. In this example, knowledge of drug dose and hypoglycaemia rates would have been informative, but are unfortunately not available. Finally, this paper highlights that differences between therapies may be apparent in 'real-life' settings that are not evident in RCTs. These differences may point to important patient or provider concerns that have an impact on treatment decisions or adherence and, hence, outcomes. Thus, extrapolating results from RCTs to the real world of clinical practice should be done with care. As the field of comparative effectiveness research matures, it is likely we will continue to face questions on how the results of RCTs and clinical effectiveness trials compare and why differences are observed. Careful interpretation of these analyses will be required to place the results in the proper clinical context and to enable providers to make the optimal use of the totality of the evidence.

Funding The author receives grant support from the US National Institutes of Health (U01DK1098245).

Duality of Interest The author declares that there is no duality of interest associated with this manuscript.

Contribution statement The author was solely responsible for drafting the manuscript and approving the final version.

\section{References}

1. DeMets DL, Califf RM (2011) A historical perspective on clinical trials innovation and leadership. Where have the academics gone? JAMA 305:713-714 
2. Ahrén B, Mathieu C, Bader G, Schweizer A, Foley JE (2014) Efficacy of vildagliptin versus sulfonylureas as add-on therapy to metformin: comparison of results from randomised controlled and observational trials. Diabetologia. doi:10.1007/s00125-014-3222-z
3. Mathieu C, Barnett AH, Brath $\mathrm{H}$ et al (2013) Effectiveness and tolerability of second-line therapy with vildagliptin vs other oral agents in type 2 diabetes: a real-life worldwide observational study (EDGE). Int J Clin Pract 67:947-956 